\title{
Wireless Energy Management System for Residential Area
}

\author{
Hong-Chan Chang and Cheng-Chien Kuo
}

\begin{abstract}
The energy management system (EMS) proposed in this paper uses the communication platform of ZigBee wireless sensor network in combination with the energy parameters measurement and the control functions of safety protection and monitor, in order to achieve the objectives of energy saving, carbon reduction and safe power usage. The system structure is consisted of the intelligent outlet module, wireless transmission module and central monitor and control module. Combining with the energy monitor and safety protection software, the system can achieve seven major functions, which are energy usage monitor, energy usage control, standby power management, circuit loop energy monitor and protection control, outlet overheat protection, electric leakage protection and neutral line over-voltage protection. The system can not only reduce energy losses and improve safety protection, but also benefit for suppressing carbon emission and mitigating the effects of global warning process and climate change.
\end{abstract}

Index Terms-Energy management, ZigBee, energy saving, smart outlet.

\section{INTRODUCTION}

The excessive usage of fossil energy has led to the threat of energy crisis. Moreover, due to worldwide industrialization, the global climate becomes abnormal and mankind is facing severe problems of existence. Therefore, besides finding clean, environment-friendly and non-exhausted new energies, it is necessary to develop appropriate energy-saving technologies, in order to effectively slow down the energy usage and alleviate the severe changes of environment.

Most of the recent researches only focus on the energy monitor and control of outlet [1]-[6]. A multi-function energy management system is still seldom. Therefore, an integrated energy management system combined with the functions of energy monitor and power control would be presented in the study. It could be used by households and office locations in any environments. Up to the high performance of easy expandability and convenience of overall system construction, it could save the unnecessary power consumption in households and improve the safety protection of household electric products so as to achieve the triple objectives on energy conservation and carbon reduction, energy monitor and safety protection.

An integrated energy management system combined with the functions of energy monitor and power control would be presented in the study. It could be used by households and office locations in any environments. Up to the high

Manuscript received April 21, 2013; revised June 8, 2013. This work were supported by the National Science Council under Project No. 99-2221-E-011-149-MY3 and NSC101-2632-E-129-001.

The authors are with the Department of Electrical Engineering, National Taiwan University of Science and Technology, Taiwan (e-mail: hcchang@mail.ntust.edu.tw, cckuo@mail.sju.edu.tw). performance of easy expandability and convenience of overall system construction, it could save the unnecessary power consumption in households and improve the safety protection of household electric products so as to achieve the triple objectives on energy conservation and carbon reduction, energy monitor and safety protection.

\section{System STRUCtURE}

Besides the function of remote wireless control, the energy management system based on ZigBee wireless transmission proposed in this paper also possesses the important functions of intelligent energy management and power usage safety protection. For the convenience of applying the developed system onto buildings, the wireless transmission function is adopted in the system so that the system could be used and installed at any location within the interior space without any need of wiring, which realizes the intelligent power usage management, monitor and safety protection at any place and effectively improves the convenience of system utilization. The system concept is as shown in Fig. 1. As shown in the figure, each piece of electric equipment is plugged onto the designed intelligent outlet module. All measured energy parameters are transmitted through the Zigbee wireless transmission module to the central monitor and control module where analysis and discriminating are made for decisions uniformly through powerful computing then commands are sent out by the wireless communication module to the intelligent outlet module to take actions accordingly. However, since some power usage safety protection functions need rather fast reaction, for instance, in the cases of electric leakage, over temperature or neutral line over voltage, power supply of the outlet needs to be disconnected instantly. Therefore these functions must make immediate decisions and take fast actions directly within the intelligent outlet module and send back the results to the central monitor and control module for display or statistic need after the actions have been completed.

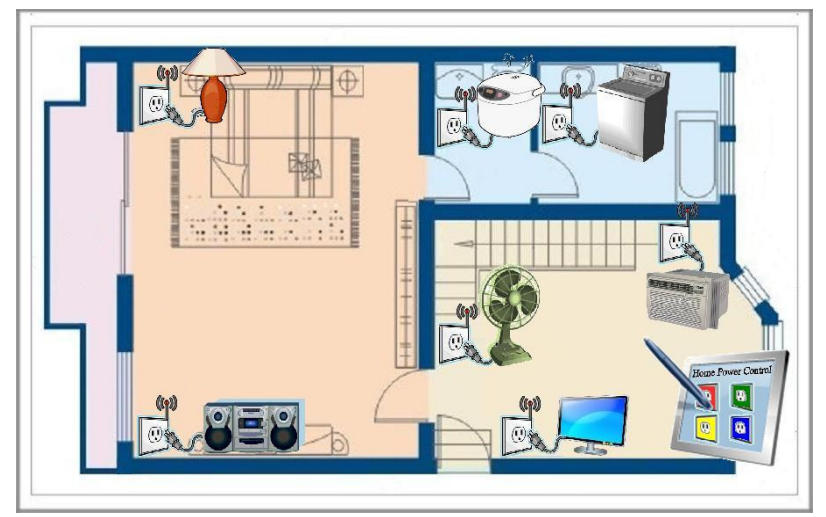

Fig. 1. Concept diagram of Energy management system

The system could flexibly set up each outlet monitor, 
circuit overload protection, standby shut-off critical value and other parameters based on power usage plan of the occupancy and usage status of each electric circuit. It also could measure the energy parameters of loads connected to each outlet by using energy detection chip; fetch the digitalized energy signals, analyze and process the digitalized signals through the microprocessor; discriminate anomalies of equipment leakage current, outlet temperature and neutral line voltage; take instant on-off circuit control appropriately; transmit the measured and processed data to the computer of central monitor and control module via Zigbee wireless transmission module to calculate and monitor the status of each outlet through the monitor program.

The energy management system proposed hereof is in a master-slave structure where the central monitor and control module, the master, is connected with each intelligent outlets, the slave, through wireless transmission. The intelligent outlet part processes many functions required for rapid responses such as outlet overheat protection, electric leakage protection and neutral line over-voltage protection. The central monitor and control module can process many functions concerned with energy management including energy usage monitor, energy usage control, standby power management and circuit loop energy monitor and protection control. The system could also customize and develop suitable energy management functions in the central monitor and control module under this structure, which could be accomplished simply by modifying the program design without changing the hardware design. So the proposed structure of the system has a very high expandability capable of coping with the need of energy management in the future implementation of the intelligent electric grid.

The proposed system could be divided into three hardware modules, namely, the intelligent outlet module, wireless transmission module and central monitor and control module. The intelligent outlet module mainly accomplishes detection of energy status, outlet overheat protection and power supply control through the over temperature detection circuit and on-off circuit, power supply control, remote control through the wireless transmission module and transmission of the detected energy signal and outlet power supply status to the central monitor and control module via wireless transmission module. The central monitor and control module would analyze and calculate the transmitted energy parameter information and display it on the system interface. The energy usage control, standby power management and circuit loop energy monitor control could be realized through integration of each intelligent outlet module by the central monitor and control module and advanced setting by the software provider. Therefore seven software functions could be attained based on the hardware structure designed in this system.

\section{SYSTEM IMPLEMENTATION}

The intelligent outlet module developed in this study includes Zigbee module, microprocessor, energy detection chip, relay, and thermistor and power supply circuits. The outlet model is of $9.2 \pm 0.1 \mathrm{~cm} \times 7.3 \pm 0.1 \mathrm{~cm}$ as shown in Fig. 2 .

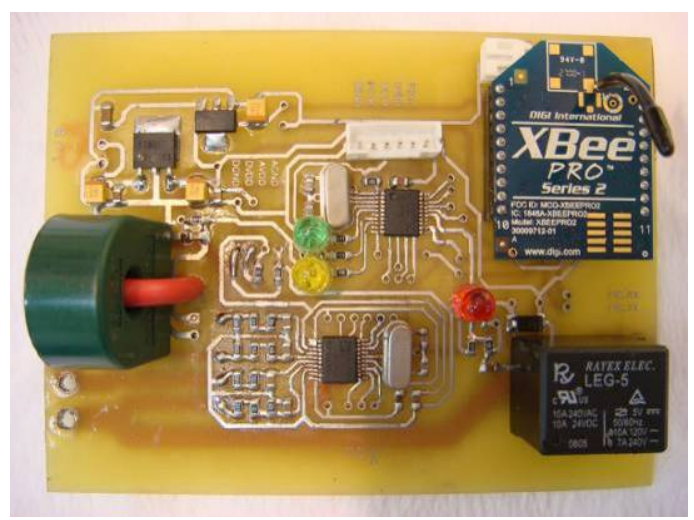

Fig. 2. The picture of intelligent outlet module

The outlet module could be put into the simple commercial outlet box made of transparent plastic and standard specifications, which could be plugged in directly for the use in old building or recessed in the wall-mounted outlet box for the use in new buildings. Fig. 3 shows the proposed intelligent outlet module placed in a commercial outlet box.

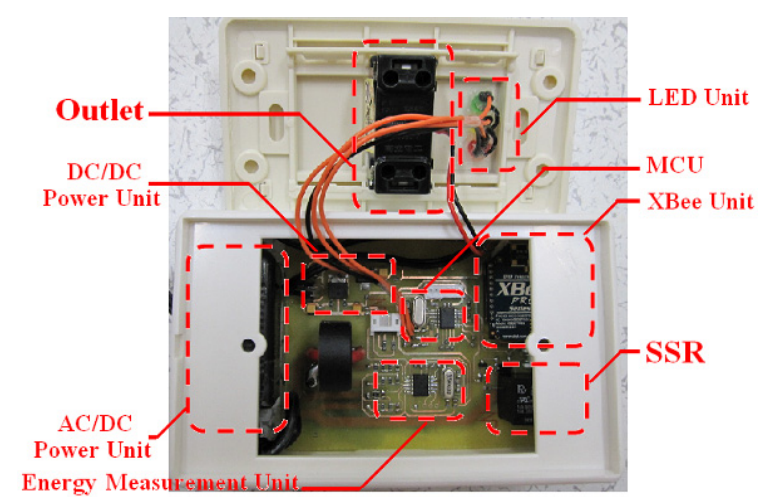

Fig. 3. The proposed intelligent outlet module placed in commercial outlet box

The proposed energy management system designed from the perspective of users could be applied in various interior environments due to its flexible configuration methods. With the simplified graphical operation interfaces, users could grasp the three major functions of energy saving, monitor and control and safety without costing too much time in installation and study. The monitor and control could be carried out through the central monitor and control module immediately after the setup of the hardware. Within the control software, the main page, each spatial page, intelligent setup, loop protection and other major and functional pages are in place and can be operated by the touch-panel method to conduct setup and operation of the system. All intelligent outlet module status are provided in the main page so that the on-off status, timer setting on-off control of each outlet and power usage status of the whole outlet systems under monitor and control could be known by the display of graphics and colors and approximate fees of overall power usage could be estimated. Fig. 4 shows the main page of EMS.

Graphics of 12 outlets are displayed in the main page representing the intelligent outlet modules in 4 spatial pages, in which 3 outlet systems are controlled respectively. Each graphic is displayed according to the setup status on each subordinate page and the back transmission state of the outlet system. The graphic is indicated in grey color when the outlet system is not energized and used and indicated in green color when the outlet system is involved in normal actions to 


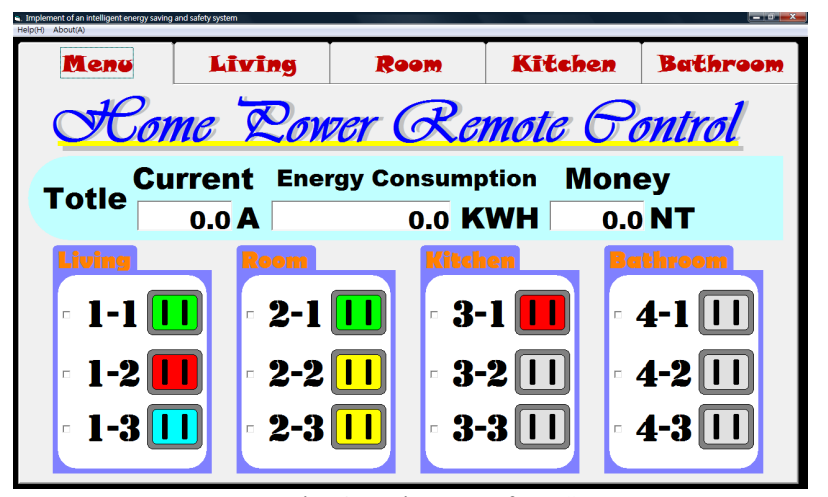

Fig. 4. Main page of EMS

inform the users that the outlet system is in normal power supply status. When the graphic is indicated in red color, it means that current outlet system is disconnected and not supplying power either because of the power supply disconnection by the user through the remote on-off control function or protection control by trip or automatic power disconnection when the outlet system detects over temperature or overload. In this case, if it is not set up or controlled by the user then the outlet system indicated in red color must be checked to verify and eliminate the failures or abnormities before the power supply is resumed. When the graphic of outlet is indicted in yellow or blue color, it means the outlet system is under timer setting on-off control by the user where the yellow indicates the timer setting off control and the blue color indicates the timer setting on control so that the users could easily discriminate the current status of each outlet system by different colors.

There are 4 preset spatial pages, namely living room, room, kitchen and bathroom. After equipment numbering of each intelligent outlet module is completed, the sequencing will be carried out based on the equipment numbering starting from the first page, for example, the living room is number as No. 1 to No.3 outlet systems, the room is numbered as No. 4 to No. 6 and the rest can be done in the small manner. Total current, total power and rough estimation of power usage fees of each space are provided in each spatial page and four kinds of energy parameters of voltage, current, power and frequency are provided for each outlet system for the users to monitor the outlet usage status all the time and take remote on-off control. The users only need to put their fingers on the central monitor and control module to turn on or off the electric equipment. Same outlet status graphics of the outlet system will also be provided on the main page so the users could identify the setup status of the outlet systems by colors. Advanced intelligent settings and loop protection selection control will be set up from this page. The spatial page is as shown in Fig. 5.

A Smart Set function selection is provided for the control area of each outlet system for users to set up the outlet system where a setup window will pop up after it is clicked and selected. Two advanced functions, namely the timer setting on-off control and standby power saving, are provided on this page. The function page of Smart Set is as shown in Fig. 6.

In the part of timer setting on-off control, users could select the on or off control when the set time of this outlet system is up. Once confirmation is made the color of the displaying graphic for the outlet system will be changed, where the yellow indicates the timer setting off control and

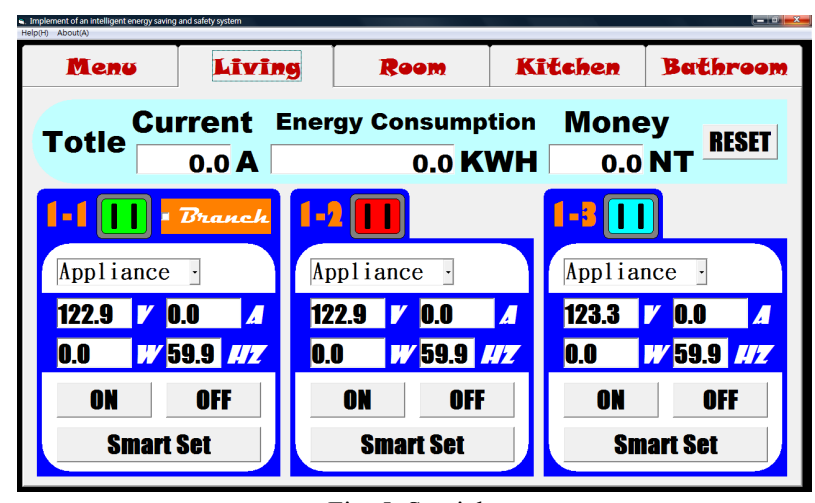

Fig. 5. Spatial page

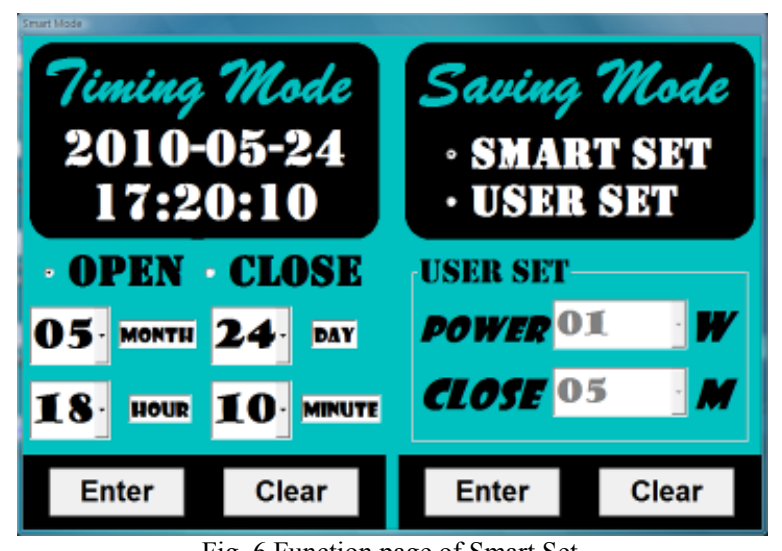

Fig. 6 Function page of Smart Set

the blue color indicates the timer setting on control so that the users could easily discriminate the current setup status by different colors. In the standby power saving part, there are two selections, namely, intelligent setup and user setup. When the electric equipment enters into the standby state after usage, the system will disconnect the outlet power supply automatically. The intelligent setup refers to the system preset values. When the power value is lower than $1 \mathrm{~W}$, the countdown of the setting time for disconnecting the outlet system will be activated automatically. The countdown time is preset as 5 minutes during which if the outlet has not been used, power supply of the outlet will be cut off. Users can also decide the user's setup selections based on actual current status of the electric equipments and their usage customs.

The Branch selection is provided for the No. 1 outlet system on each spatial page and shall be implemented in cooperation with the outlet system hardware setup where a setup page will pop up after it is clicked and selected. The selection of intelligent trip is preset by the system. When the No. 1 outlet system on the spatial page detects that the rest of the outlet systems exceed the preset current protection value, power supply for the outlet system that is finally plugged into the electric equipment will be disconnected. Users can also set up otherwise cut-off sequence of the outlet systems to avoid power disconnection of the key equipments. Function page of Branch is as shown in Fig. 7.

The Help function is included in the tools of the system so that users could hit it and look for explanation in case of questions for the system.

There are 3 LED lights on the intelligent outlet module panel, through which the users could discriminate the operation status of the outlet immediately. 


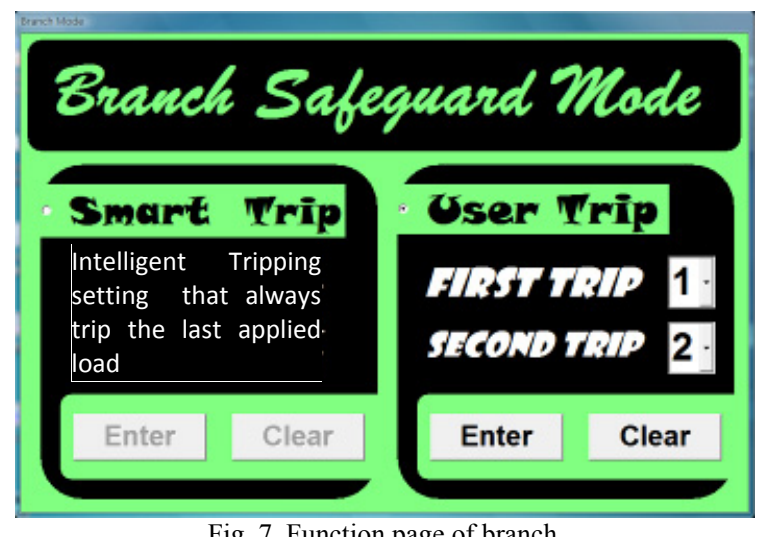

Fig. 7. Function page of branch

When the red light is on, power supply of the outlet system is disconnected. When the yellow light and red light are both on, the power supply is stopped due to over temperature. When the green light and red light are both on, the electric leakage protection is activated. The reset function panel is provided in the outlet system to be selected by the users upon their choice based on the environment and customs.

\section{CONCLUSION}

In this paper, a set of energy management system has been successfully accomplished, which primarily achieves the detection of the outlet power supply status during the usage of electric equipment loads and ordinary times with its automation and safety being further improved by the microprocessor and other surrounding circuits. Combined with ZigBee wireless transmission function, it offers convenience for remote monitoring of the status of each monitoring point, effectively elevates the quality and convenience of power usage and smoothly improves the situation where power usage was unknown to traditional outlets thus users are able to grasp the power usage status through displayed values, take correct protection to the lines to avoid overload and prevent from property losses and personal injuries due to anomalies or overload of the electric equipments and has certain socioeconomic benefits and vast development prospects. Energy monitor is the most effective method of energy saving and safety protection. Generally, when the electric equipments are not in usage, most of the power consumption is standby power consumption that could be eliminated. Without the aid of observation through the monitor and control system, the accumulated power consumption during electric equipment standby period is hard to be perceived. Also, the continuous loading on the same loop under unknown circumstance would result in not only unnecessary energy loss but also major hazards. By using the energy monitor system and automatic protection lines, the overload could be prevented. Disconnecting the standby electric equipments automatically could ensure the power usage safety and bring in benefits in energy saving. The feature of ZigBee in supporting to a large number of nodes is benefit for its future expansion where the newly migrated intelligent outlet nodes could combine with the original system immediately. Its convenience in setup and expansion meets the requirements of various power usage environments. A graphic monitor and control system is constructed by the central monitor and control module by using Visual Basic software. With the combination of the touch panel computer setup and easy operation interfaces, it is easy for operation by anybody and realizes the all-time monitor and control in terms of energy saving and safety.

The application of the energy management system has improved the quality of electric usage of the households and offices through which the power could be reviewed through values and controlled at any time and elevated the level of power usage safety. Therefore, it not only contributes to the energy conservation and carbon reduction but also improves living quality and safety.

\section{REFERENCES}

[1] G. Song, F. Ding, W. Zhang, and A. Song, “A wireless power outlet system for smart homes," IEEE Transactions on Consumer Electronics, vol. 54, no. 4, pp. 1688-1691, November 2008.

[2] C. H. Lien, C. H. Lin, Y. W. Bai, M. F. Liu, and M. B. Lin, "Remotely controllable outlet system for home power management," IEEE Tenth International Symposium on Consumer Electronics, pp. 1-6, 2006.

[3] C. H. Lien, Y. W. Bai, and M. B. Lin, "Remote-controllable power outlet system for home power management," IEEE Transactions on Consumer Electronics, vol. 53, no. 4, pp. 1634-1641, November 2007.

[4] C. H. Lien, H. C. Chen, Y. W. Bai, and M. B. Lin, "Power monitoring and control for electric home appliances based on power line communication," IEEE Instrumentation and Measurement Technology Conference, pp. 2179-2184, May 2008.

[5] J. Han, H. Lee, and K. Park, "Remote-controllable and energy-saving room architecture based on ZigBee communication," IEEE Transactions on Consumer Electronics, vol. 55, no. 1, pp. 264-268, February 2009.

[6] W. S. Lee and S. H. Hong, "KNX - ZigBee Gatway for home automation," IEEE International Conference on Automation Science and Engineering, pp. 750-755, August 2008.

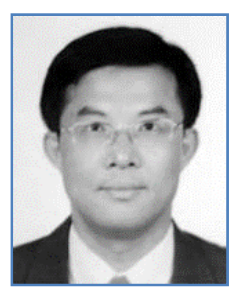

Hong-Chan Chang was born in Taipei, Taiwan on March 5, 1959. He received the B.S., M.S., and Ph.D. degrees, all in electrical engineering, from National Cheng Kung University in 1981, 1983, and 1987 respectively. In August 1987, he joined National Taiwan University of Science and Technology, Taipei, Taiwan, as a faculty member. He is presently a Professor. His major areas of research include power system stability, reliability, and application of artificial intelligence to power systems.

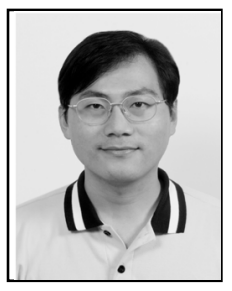

Cheng-Chien Kuo was born on August 9, 1969, in YunLin, Taiwan, where he received his B.S., M.S., and $\mathrm{Ph} . \mathrm{D}$. degrees all from the National Taiwan University of Science and Technology (NTUST) in 1991, 1993, and 1998, respectively. He has been with St. John's University since 1994, where he is currently a Professor in the Department of Electrical Engineering. His research interests include energy management system, microprocessor application system, Smart grid application 